\title{
Pharmaceutical care for older adults in Brazil: a systematic review
}

\section{Cuidados farmacêuticos para idosos no Brasil: uma revisão sistemática}

Recebido em: 13/03/2021 Aceito em: $02 / 06 / 2021$
Maria Olívia Barboza ZANETTI'; ; Ana Flávia Miguel dos SANTOS²; Débora Ferreira SANTOS ${ }^{2}$; Leonardo Régis Leira PEREIRA ${ }^{3}$ ${ }^{1}$ Centro Universitário Barão de Mauá - Unidade Central. R. Ramos de Azevedo, 423, Jardim Paulista, CEP 14090-062. Ribeirão Preto, SP, Brasil. ${ }^{2}$ Faculdade de Ciências da Saúde de Barretos Dr. Paulo Prata. Av. Loja Maçônica Renovadora 68, $N^{\circ}$ 100, CEP 14785-002. Barretos, SP, Brasil.

${ }^{3}$ Faculdade de Ciências Farmacêuticas de Ribeirão Preto, Universidade de São Paulo (USP). Av. do Café, s/n, Vila Monte Alegre, CEP 14040-900. Ribeirão Preto, SP, Brasil. E-mail:marolzanetti@gmail.com

\section{ABSTRACT}

Considering the specific requirements of the older adults in the use of drugs and the opportunity for the clinical pharmacist to contribute to these patients, this review aimed to identify studies that have evaluated the results of pharmaceutical care for older adults in Brazil. We conducted a systematic review of the studies that answered the following guiding question: "What pharmaceutical care outcomes in older adult patients are found in Brazil?". The search strategy was applied to the following databases in February 2019: PubMed, CENTRAL, BVS, and LILACS. Observational or experimental studies describing the results of pharmaceutical care in older adults in Brazil at any level of healthcare and related to the monitoring of any health problem were included. The quality of the included publications was assessed using the Downs and Black checklist. Of the 5080 studies that were identified, 11 were included. The results could summarize evidences supporting pharmaceutical care for older adults, regardless of place of attendance, health problem, or level of health care. The experiences of the Brazilians have contributed to the improvement of clinical and humanistic outcomes, such as reduction in blood pressure, glycemic index, lipid levels, anthropometric measurements, and polypharmacy; resolution of drugs related problems; and improvement in adherence and quality of life. One publication also reported economic outcomes (ICER per QALY gained). Although the number of articles obtained was small, they reported unanimously beneficial results. Pharmacist's training in healthcare service is essential for expanding pharmaceutical care for this population group.

Keywords: elderly; health services for the elderly; pharmaceutical services; pharmaceutical care.

\section{RESUMO}

Considerando as especificidades da população idosa em relação ao uso de medicamentos e a oportunidade de contribuição do farmacêutico clínico, esta revisão teve como objetivo identificar estudos que avaliaram os 
resultados do cuidado farmacêutico direcionado a idosos brasileiros. Foi conduzida uma revisão sistemática de estudos brasileiros que respondiam à questão norteadora: "Quais são os resultados do cuidado farmacêutico em idosos no Brasil?". A estratégia de busca foi aplicada nas bases de dados PubMed, CENTRAL, BVS e LILACS em fevereiro de 2019. Foram incluídos estudos observacionais ou experimentais que descreviam os resultados do cuidado farmacêutico em pacientes idosos no Brasil, em qualquer nível de atenção à saúde e no acompanhamento de qualquer problema de saúde. Foi utilizado o instrumento Downs and Black para avaliar a qualidade das publicações. Foram identificados 5080 estudos, sendo onze considerados adequados para inclusão na revisão. Os resultados conseguiram sumarizar evidências que apoiam a realização do cuidado farmacêutico voltado aos idosos, independentemente do local de atendimento, doença, ou nível de Atenção à Saúde. As experiências brasileiras contribuíram para a melhoria de desfechos clínicos e humanísticos, com redução dos valores pressóricos, glicêmicos, lipídicos e antropométricos, redução de polifarmácia, resolução de problemas relacionados à farmacoterapia e melhoria da adesão e da qualidade de vida. Uma publicação também relatou a contribuição para desfechos econômicos (ganho em ICER per QALY). Apesar do pequeno número de artigos encontrados, os resultados benéficos foram unânimes. Para que o cuidado farmacêutico nesta população seja amplificado, é fundamental a capacitação dos farmacêuticos.

Palavras-chave: idoso; serviços de saúde para idosos; assistência farmacêutica; atenção farmacêutica.

\section{INTRODUCTION}

As a result of improvements in health and sanitation in the last century, we have observed a worldwide increase in life expectancy with lower birth rates, causing a significant increase in the aging population (1).

In Brazil, the scenario is no different; the projections of the Brazilian Institute of Geography and Statistics indicate that by 2031, the number of older adult individuals will be equal to that of young people, and by 2055 , there will be 34.8 million young and 70.3 million older adult people in the country (2).

Currently, in Brazil, we face a triple burden of chronic non-communicable diseases, conditions from external causes (such as violence and traffic accidents), and infectious diseases (such as tuberculosis, yellow fever, and acquired immunodeficiency syndrome). The epidemiological transition is coupled with the demographic transition, which explains the prevalence of chronic diseases. Demographic changes related to aging can transform populations' health profile, characterized by an increased burden of chronic non-communicable diseases, a higher number of comorbidities, polypharmacy, and the need for continuous medical attention, particularly in more advanced age groups (3). In addition, older adults present several physiological, age-related changes that can reduce their functional capacity and make it difficult for the body to respond normally to injuries and diseases. This process is known as a geriatric syndrome and demands specialized and critical care for this population more often $(4,5)$.

The geriatric syndrome can modify the body's response to drugs, affecting both pharmacokinetics and pharmacodynamics. The possibility of damage induced by pharmacotherapy increases considerably even when the drug is employed in the correct dosages and indications $(3,4,6)$. This fact is why the use of some drugs, such as anticholinergics, may cause more risks than benefits and, thus, are known as potentially inappropriate drugs for the elderly $(7,8)$.

All these factors lead to an increase in the incidence of Drug-Related Problems (DRP) in elderly patients (3). Therefore, choosing a pharmacotherapeutic regimen should be even more meticulous, aiming at preventing adverse events and maintaining the quality of life in this age group. Previous studies have shown that the inclusion of a clinical pharmacist in the health care team can contribute to the care of this population, especially 
in reducing DRP, providing health education, and improving pharmacotherapy adherence $(3,9)$. However, there is not a large study carried out in Brazil that describes the impact of the clinical pharmacist in the care of older adults. Therefore, to obtain a national panorama, it is necessary to review Brazilian publications investigating this scenario.

Considering the specificities of the older adult population regarding the use of drugs and the opportunity for the contribution of the pharmacist, this review aimed to identify studies that reported the results of pharmaceutical care for older adult patients in Brazil, answering the question "What pharmaceutical care outcomes in older adult patients are found in Brazil?"

\section{METHOD}

This study is a systematic review conducted following the Preferred Reporting Items for Systematic Reviews and Meta-Analyses guideline (PRISMA) (10). We selected the publications that responded to the guiding question, "What pharmaceutical care outcomes in older adult patients are found in Brazil?"

Observational or experimental studies describing the results of pharmaceutical care in older adult patients in Brazil at any level of healthcare and in the treatment/follow-up of any health problem were included, regardless of the year of publication. A minimum pharmacotherapeutic follow-up period has not been determined. The established exclusion criteria were as follows: articles describing the results of pharmaceutical care without separating the results by age group; articles that have described the results of services of multi-professional teams in the care of older adult patients but have not presented the activities and results of pharmaceutical care separately; publications only theoretically describing pharmaceutical care in older adult populations; dissertations or theses, expert comments or opinions, study protocols, editorials, news, and abstracts published in congress proceedings; articles written in languages other than English, Portuguese, or Spanish; publications without complete text available.
The formulation of the search strategy sought to incorporate terms that referred to the guiding question and were planned according to the acronym PICOS (Population: older adult patients; Intervention: pharmaceutical care/clinical services performed by pharmacists; Comparison: absence of pharmaceutical care/clinical pharmacist; Outcomes: the outcomes were not defined at this stage of the search so as not to assign an undesired specificity at the time; Study design: observational or experimental studies) (11). The search for and association of such terms in the Medical Subjects Headings dictionary of the PubMed database resulted in the main search strategy (Table 1), which was adapted to the other researched databases: CENTRAL (The Cochrane Central Register of Controlled Trials The Cochrane Library), BVS (Virtual Health Library), and LILACS (Scientific and Technical Literature of Latin America and the Caribbean). No filters were added to the search strategy, such as languages, country, article design, or population, not to miss potential articles of interest. The search was conducted in February 2019.

The web application "Rayyan - a web and mobile app for systematic reviews" assisted in selecting the publications for inclusion (12). Initially, duplicate articles in the databases were removed. To screen the articles of interest for full reading, a critical analysis of the title and abstract of the publications according to the pre-established inclusion and exclusion criteria was initiated. Two researchers (AFMS and DFS) were selected to perform the step as mentioned above independently, reducing biases during screening. Disagreements between the two researchers were solved through discussion and consensus, and in cases of persistent disagreement, a third researcher (MOBZ) was responsible for the decision.

The remaining publications were then read in their entirety by the same researchers who defined which articles met the inclusion criteria and would be incorporated in the review. In addition, a manual search was performed using the reference list of the selected articles to search for other relevant publications that the initial strategy might not have obtained. All publications selected for full reading had the full text available and were either in Portuguese or English. 
Table 1. Description of the search strategy employed, with descriptors and keywords.

\begin{tabular}{|c|c|c|}
\hline $\begin{array}{l}\text { Search } \\
\text { strategy }^{1}\end{array}$ & $\begin{array}{l}\text { Subject descriptor } \\
\text { (MeSH terms) }\end{array}$ & Relevant keywords \\
\hline 1 & $\begin{array}{l}\text { "Aged"; "Health Services for } \\
\text { the Aged" }\end{array}$ & $\begin{array}{l}\text { "Aged" } O R \text { "Older adult" } O R \text { "Health Services for the Aged" } O R \text { "Geriatric Health } \\
\text { Services" } O R \text { "Health Services for the Older adult" } O R \text { "Health Services, Geriatric" } \\
O R \text { "Geriatric Health Service" OR "Health Service, Geriatric" } O R \text { "Service, Geriatric } \\
\text { Health" } O R \text { "Services, Geriatric Health" OR "Health Services for Aged" }\end{array}$ \\
\hline 2 & $\begin{array}{l}\text { "Pharmacy Service, Hospital"; } \\
\text { "Pharmaceutical Services"; } \\
\text { "Pharmacists" }\end{array}$ & $\begin{array}{l}\text { "Pharmacy Service, Hospital"; "Hospital Pharmaceutical Service"; "Hospital } \\
\text { Pharmaceutical Services"; "Pharmaceutical Services, Hospital"; "Services, } \\
\text { Hospital Pharmaceutical"; "Pharmaceutical Service, Hospital"; "Pharmaceutic } \\
\text { Service, Hospital"; "Hospital Pharmacy Services"; "Pharmacy Services, Hospital"; } \\
\text { "Services, Hospital Pharmacy"; "Service, Hospital Pharmacy"; "Hospital } \\
\text { Pharmacy Service"; "Hospital Pharmaceutic Service"; "Hospital Pharmaceutic } \\
\text { Services"; "Pharmaceutic Services, Hospital"; "Services, Hospital Pharmaceutic"; } \\
\text { "Service, Hospital Pharmaceutic"; "Service, Hospital Pharmaceutical"; } \\
\text { "Pharmacy Service, Clinical"; "Service, Clinical Pharmacy"; "Clinical Pharmacy } \\
\text { Services"; "Pharmacy Services, Clinical"; "Services, Clinical Pharmacy"; "Clinical } \\
\text { Pharmacy Service"; "Pharmaceutical Services"; "Services, Pharmaceutic"; } \\
\text { "Services, Pharmacy"; "Pharmaceutic Services"; "Pharmaceutic Service"; } \\
\text { "Service, Pharmaceutic"; "Services, Pharmaceutical"; "Pharmaceutical Service"; } \\
\text { "Service, Pharmaceutical"; "Pharmacy Services"; "Pharmacy Service"; "Service, } \\
\text { Pharmacy"; "Pharmaceutical Care"; "Care, Pharmaceutical"; Pharmacists; } \\
\text { Pharmacist; "Clinical Pharmacists"; "Clinical Pharmacist"; "Pharmacist, } \\
\text { Clinical"; "Pharmacists, Clinical" }\end{array}$ \\
\hline
\end{tabular}

Finally, a form for extracting the data of interest was developed according to the Cochrane Reviewer's Handbook (13), and an article was randomly selected to test its applicability. We extract the following variables: author, year of publication, the origin of the study, title, study design, sample, place and time of pharmaceutical follow-up, health problem, aim, pharmaceutical clinical services performed, and outcomes. All data were extracted according to the original article authors' description; the authors made no simplification or assumptions to avoid misinterpretation. After adjusting the form, data were extracted from each included article, which were also extracted in duplicates by two researchers (AFMS and DFS), and the discrepancies were resolved by the third researcher (MOBZ). There was no need to contact the authors of the included articles to obtain additional information.

The modified Downs and Black checklist for measuring study quality was used to evaluate the articles' methodological quality, external and internal validity, and statistical power $(14,15)$. This tool allowed the evaluation of the observational and interventional studies and presented a maximum score of 28 points, classifying the methodological quality as excellent (24-28 points), good (19-23 points), average (14-18 points), or bad ( $<14$ points).

\section{RESULTS AND DISCUSSION}

The application of the search strategy obtained a total of 5679 publications following title and abstract screening and one publication through searching the reference lists; 599 were duplicates, 5081 publications were assessed for eligibility, and 13 were selected by the researchers (AFMS and DFS) for a full reading. The reasons for exclusion when screening the title and abstract were: title/ abstract not matching the research question ( $\mathrm{n}=$ 5041), results of services of multi-professional teams in the care of elderly patients $(n=13)$, news $(n=10)$, experts comments or opinions $(n=3)$, theoretically describing pharmaceutical care in elderly populations $(\mathrm{n}=1)$. 
Figure 1. Flowchart of the process of article selection for the systematic review.

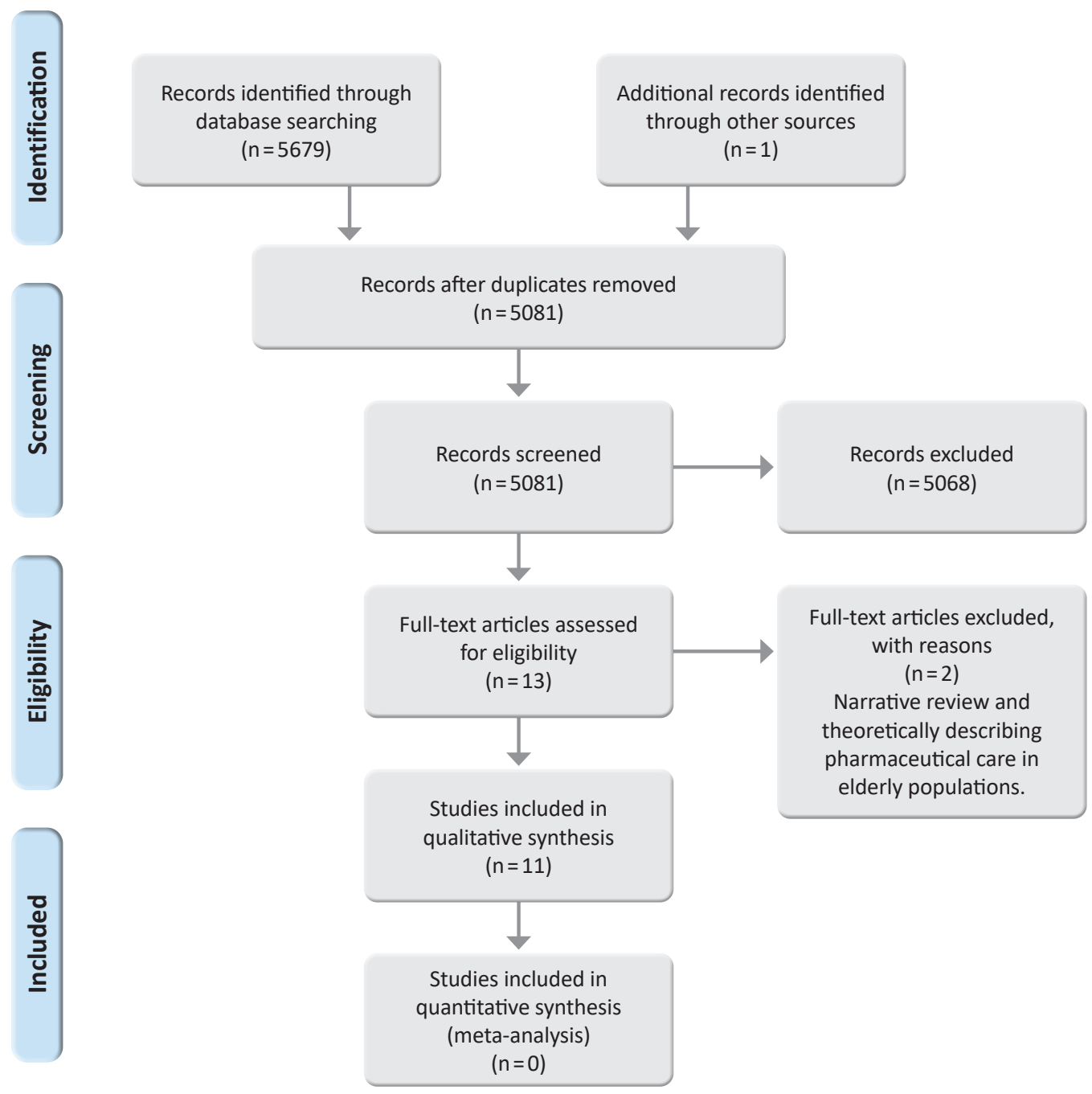

After full reading, there was a disagreement regarding the inclusion of two publications in the review; these publications were excluded after analysis by the third researcher (MOBZ) because one of them was a narrative review and the other described theoretically pharmaceutical care in elderly populations. Finally, 11 articles were included.

Figure 1 illustrates the selection process for the studies used in this review.In the studies included in the systematic review, clinical services performed by the pharmacists in seven studies were offered in the basic health units (16-22), one in a community pharmacy (23), one in patient's home (24), one in a geriatric nursing home (25), and one in a hospital (5).
Table 2 presents the quality scores of the articles included. Three publications received a score corresponding to good methodological quality $(18,19,22)$, five manuscripts corresponding to medium quality $(16,17,20,21,23)$, and three corresponding to poor quality $(5,24,25)$. None of the studies obtained the classification of excellence. Regarding the type of health problems managed by the pharmacists, 10 publications assessed hypertension (16-25); in three cases, older adult patients had to present a diagnosis of hypertension and/ or diabetes mellitus to receive pharmacotherapy monitoring $(18,19,22)$. One study included older adult people who needed critical care regardless of the health problem presented (5). 
Table 2. Presentation of the methodological quality of the articles included in the review $(n=11)$, according to the tool Downs \& Black.

\begin{tabular}{|c|c|c|c|c|c|c|c|c|c|c|c|c|c|c|c|c|c|c|c|c|c|c|c|c|c|c|c|c|}
\hline \multicolumn{29}{|c|}{ Downs \& Black appraisal criteria } \\
\hline \multirow[t]{2}{*}{ Author, year } & \multicolumn{10}{|c|}{ Reporting } & \multicolumn{3}{|c|}{$\begin{array}{l}\text { External } \\
\text { validity }\end{array}$} & \multicolumn{7}{|c|}{ Internal validity - bias } & \multicolumn{6}{|c|}{$\begin{array}{l}\text { Internal validity - } \\
\text { confounding } \\
\text { (selection bias) }\end{array}$} & \multirow{2}{*}{\begin{tabular}{|c|} 
Power \\
27 \\
\end{tabular}} & \multirow[t]{2}{*}{ Score } \\
\hline & 1 & 2 & 3 & 4 & 5 & 6 & 7 & 8 & 9 & 10 & 11 & 12 & 13 & 14 & 15 & 16 & 17 & 181 & 192 & 20 & 21 & 22 & 23 & 24 & 252 & 26 & & \\
\hline Aguiar et al., 2012 & $\checkmark$ & $\checkmark$ & $\checkmark$ & $\checkmark$ & $x$ & $\checkmark$ & $\checkmark$ & $\mathrm{x}$ & $x$ & $\checkmark$ & $x$ & $x$ & $\checkmark$ & $x$ & $x$ & $\checkmark$ & $\checkmark$ & $\checkmark$ & $\checkmark \checkmark$ & $\checkmark$ & $x$ & $x$ & $x$ & $x$ & $x$ & $x$ & $\checkmark$ & 14 \\
\hline Brito et al., 2009 & $\checkmark$ & $x$ & $\checkmark$ & $\checkmark$ & $x$ & $\checkmark$ & $\checkmark$ & $x$ & $\checkmark$ & $x$ & $x$ & $x$ & $\checkmark$ & $x$ & $x$ & $\checkmark$ & $\checkmark$ & $\mathrm{x}$ & $\checkmark x$ & $x$ & $x$ & $\mathrm{x}$ & $\mathrm{x}$ & $\mathrm{x}$ & $\mathrm{x}$ & $\checkmark$ & $\mathrm{x}$ & 11 \\
\hline Lyra-Júnior et al., 2005 & $\checkmark$ & $\checkmark$ & $\checkmark$ & $\checkmark$ & $x$ & $\checkmark$ & $\checkmark$ & $x$ & $\checkmark$ & $x$ & $x$ & $x$ & $\checkmark$ & $x$ & $x$ & $\checkmark$ & $\checkmark$ & $\checkmark$ & $\checkmark \checkmark$ & $\checkmark$ & $\mathrm{x}$ & $x$ & $\mathrm{x}$ & $x$ & $\mathrm{x}$ & $\checkmark$ & $x$ & 14 \\
\hline Lyra-Júnior et al., 2007a & $\checkmark$ & $\checkmark$ & $\checkmark$ & $\checkmark$ & $x$ & $\checkmark$ & $\checkmark$ & $\mathrm{x}$ & $\checkmark$ & $\checkmark$ & $x$ & $x$ & $\checkmark$ & $x$ & $x$ & $\checkmark$ & $\checkmark$ & $\checkmark$ & $\checkmark \checkmark$ & $\checkmark$ & $x$ & $x$ & $\mathrm{x}$ & $x$ & $\mathrm{x}$ & $\checkmark$ & $\checkmark$ & 16 \\
\hline Lyra-Júnior et al., 2007b & $\checkmark$ & $\checkmark$ & $\checkmark$ & $\checkmark$ & $x$ & $\checkmark$ & $\checkmark$ & $x$ & $\checkmark$ & $x$ & $x$ & $x$ & $\checkmark$ & $x$ & $x$ & $\checkmark$ & $\checkmark$ & $\checkmark$ & $\checkmark \checkmark$ & $\checkmark$ & $x$ & $x$ & $\mathrm{x}$ & $x$ & $x$ & $\checkmark$ & $\checkmark$ & 15 \\
\hline Lyra-Júnior, Marcellini, Pelá, 2008 & $\checkmark$ & $\checkmark$ & $\checkmark$ & $\checkmark$ & $x$ & $\checkmark$ & $\checkmark$ & $x$ & $\checkmark$ & $\checkmark$ & $x$ & $x$ & $\checkmark$ & $x$ & $x$ & $\checkmark$ & $\checkmark$ & $\checkmark$ & $\checkmark \checkmark$ & $\checkmark$ & $x$ & $x$ & $x$ & $x$ & $x$ & $\checkmark$ & $\checkmark$ & 16 \\
\hline Obreli-Neto et al., 2011a & $\checkmark$ & $\checkmark$ & $\checkmark$ & $\checkmark$ & $x$ & $\checkmark$ & $\checkmark$ & $x$ & $\checkmark$ & $\checkmark$ & $\checkmark$ & $\checkmark$ & $\checkmark$ & $x$ & $x$ & $\checkmark$ & $\checkmark$ & $\checkmark$ & $\checkmark \checkmark$ & $\checkmark$ & $\checkmark$ & $\checkmark$ & $\checkmark$ & $x$ & $x$ & $\checkmark$ & $\checkmark$ & 21 \\
\hline Obreli-Neto et al., 2011b & $\checkmark$ & $\checkmark$ & $\checkmark$ & $\checkmark$ & $x$ & $\checkmark$ & $\checkmark$ & $x$ & $\checkmark$ & $\checkmark$ & $\checkmark$ & $\checkmark$ & $\checkmark$ & $\mathrm{x}$ & $x$ & $\checkmark$ & $\checkmark$ & $\checkmark$ & $\checkmark \checkmark$ & $\checkmark$ & $\checkmark$ & $\checkmark$ & $\checkmark$ & $x$ & $x$ & $\checkmark$ & $\checkmark$ & 21 \\
\hline Obreli-Neto et al., 2015 & $\checkmark$ & $\checkmark$ & $\checkmark$ & $\checkmark$ & $\mathrm{x}$ & $\checkmark$ & $\checkmark$ & $x$ & $\checkmark$ & $\checkmark$ & $\checkmark$ & $\checkmark$ & $\checkmark$ & $x$ & $\mathrm{x}$ & $\checkmark$ & $\checkmark$ & $\checkmark$ & $\checkmark v$ & $\checkmark$ & $\checkmark$ & $\checkmark$ & $\checkmark$ & $\mathrm{x}$ & $\mathrm{x}$ & $\checkmark$ & $\checkmark$ & 21 \\
\hline Reinhardt et al., 2012 & $\checkmark$ & $\checkmark$ & $\checkmark$ & $\checkmark$ & $\mathrm{x}$ & $\checkmark$ & $\checkmark$ & $\mathrm{x}$ & $\mathrm{x}$ & $\checkmark$ & $\mathrm{x}$ & $\mathrm{x}$ & $\checkmark$ & $\mathrm{x}$ & $\mathrm{x}$ & $\checkmark$ & $x$ & $\checkmark$ & $\checkmark \checkmark$ & $\checkmark$ & $\mathrm{x}$ & $\mathrm{x}$ & $\mathrm{x}$ & $\mathrm{x}$ & $\mathrm{x}$ & $\mathrm{x}$ & $x$ & 12 \\
\hline Viana, Arantes, Ribeiro, 2017 & $\checkmark$ & $\mathrm{x}$ & $\checkmark$ & $\checkmark$ & $\mathrm{x}$ & $\checkmark$ & $\checkmark$ & $\mathrm{x}$ & $\checkmark$ & $\mathrm{x}$ & $\mathrm{x}$ & $\mathrm{x}$ & $\checkmark$ & $\mathrm{x}$ & $\mathrm{x}$ & $\checkmark$ & $\mathrm{x}$ & $\mathrm{x}$ & 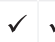 & $\checkmark$ & $\mathrm{x}$ & $\mathrm{x}$ & $\mathrm{x}$ & $\mathrm{x}$ & $\mathrm{x}$ & $\checkmark$ & $\mathrm{x}$ & 11 \\
\hline
\end{tabular}

$\checkmark$ : Yes; $\mathbf{X}$ : No or unable to determine.

1. Hypotheses/aims/objectives clearly stated; 2 . Main outcome measures clearly described; 3 . Characteristics of patients/subjects clearly described; 4. Interventions of interest clearly described; 5 . Distribution of principal confounders in each group clearly described; 6 . Main findings clearly described; 7. Estimates of random variability in the data provided; 8 . Important adverse events reported; 9 . Characteristics of patients lost to follow-up described; 10. Actual probability values reported; 11 . Participants approached representative of entire population; 12 . Participants recruited representative of entire population; 13. Staff, places, and facilities representative of majority of population; 14 . Blinding of study subjects; 15 . Blinding of assessors; 16. Data based on data-dredging clearly stated; 17 . Adjustment of different length of follow-up or duration between case and control;

18. Appropriate statistical tests used; 19 . Compliance to intervention reliable; 20. Main outcome measure reliable and valid; 21 . Intervention groups or case-controls recruited from same population; 22 . Intervention groups or case-controls recruited at the same time; 23 . Study subjects randomized to the interventions; 24 . Was concealed randomization to allocation undertaken; 25 . Adequate adjustment made in the analysis of confounders; 26. Patient losses accounted for; 27 . Sufficiently statistical power.

Concerning the place of development of the clinical activities, eight types of research were developed in Southeast Brazil, four in Ribeirão Preto - São Paulo $(16,17,20,21)$, three in Salto Grande - São Paulo $(18,19,22)$, and one in São Paulo - São Paulo (5). Two surveys were conducted in Northeast Brazil, in the municipality of Aracajú - Sergipe $(23,24)$, and one in the South, in Novo Hamburgo - Rio Grande do Sul (25).

Following the complete reading of the articles, we could see that the four papers from Ribeirão Preto, referred to the same research, which resulted in articles with different approaches. A similar data presentation was observed with the three articles from Salto Grande.

The activities performed by the clinical pharmacists included the following: pharmacotherapeutic follow-up, pharmacotherapy review, educational activities with groups of patients, assessment of pharmacotherapy adherence, and therapeutic drug monitoring. In all the cases, there was pharmaceutical consultation, associated or without other activities.

Regarding the applicability of the clinical pharmacist's performance in the care of older adult patients, there was unanimity in terms of positive references. This profession proved to be necessary and beneficial for both primary care and in hospital environment. In addition, two selected articles proved that the pharmacist is not only useful clinically but also useful in terms of humanistic gains by improving the quality of life of older adult patients and satisfaction with healthcare $(16,20)$. Pharmaceutical care also demonstrated to be cost-effective (22). Table 3 and Table 4 present the summary of publications included in this review. 
Table 3. Characteristics of the included articles $(n=11)$.

\begin{tabular}{|c|c|c|c|c|c|}
\hline $\begin{array}{l}\text { Author, year } \\
\text { (origin) }\end{array}$ & Title & Study design & Sample $(n)$, place & $\begin{array}{l}\text { Time of } \\
\text { follow up }\end{array}$ & $\begin{array}{l}\text { Health } \\
\text { problem }\end{array}$ \\
\hline $\begin{array}{l}\text { Aguiar et al., } \\
2012 \\
\text { (Aracajú, Sergipe) }\end{array}$ & $\begin{array}{l}\text { Pharmaceutical care program for older } \\
\text { adult patients with uncontrolled } \\
\text { hypertension. }\end{array}$ & $\begin{array}{l}\text { Interventional } \\
\text { study }\end{array}$ & $\begin{array}{l}51 \text { (between } 60 \text { and } \\
75 \text { years old), } \\
\text { Community pharmacy }\end{array}$ & 10 months & Hypertension \\
\hline $\begin{array}{l}\text { Brito et al., } \\
2009 \\
\text { (Aracajú, Sergipe) }\end{array}$ & $\begin{array}{l}\text { Effect of a pharmacotherapeutic } \\
\text { management program in a group of } \\
\text { older adult people with hypertension } \\
\text { in Aracajú-Sergipe. }\end{array}$ & $\begin{array}{l}\text { Interventional } \\
\text { study }\end{array}$ & $\begin{array}{l}30 \text { (between } 60 \text { and } \\
75 \text { years old), } \\
\text { patient's home }\end{array}$ & 20 months & Hypertension \\
\hline $\begin{array}{l}\text { Lyra-Júnior et al., } \\
2005 \\
\text { (Ribeirão Preto, } \\
\text { São Paulo) }\end{array}$ & $\begin{array}{l}\text { Satisfaction as an outcome of a } \\
\text { pharmaceutical care program for older } \\
\text { adult in Ribeirão Preto - São Paulo } \\
\text { (Brazil). }\end{array}$ & $\begin{array}{l}\text { Interventional } \\
\text { study }\end{array}$ & $\begin{array}{l}30 \text { (between } 60 \text { and } \\
75 \text { years old), Basic } \\
\text { Health Unit }\end{array}$ & 12 months & Hypertension \\
\hline $\begin{array}{l}\text { Lyra-Júnior et al., } \\
\text { 2007a } \\
\text { (Ribeirão Preto, } \\
\text { São Paulo) }\end{array}$ & $\begin{array}{l}\text { Impact of Pharmaceutical Care } \\
\text { interventions in the identifi cation and } \\
\text { resolution of drug-related problems } \\
\text { and on quality of life in a group of } \\
\text { older adult outpatients in Ribeirão } \\
\text { Preto (São Paulo), Brazil. }\end{array}$ & $\begin{array}{l}\text { Interventional } \\
\text { study }\end{array}$ & $\begin{array}{l}30 \text { (between } 60 \text { and } \\
75 \text { years old), Basic } \\
\text { Health Unit }\end{array}$ & 12 months & Hypertension \\
\hline $\begin{array}{l}\text { Lyra-Júnior et al., } \\
2007 b \\
\text { (Ribeirão Preto, } \\
\text { São Paulo) }\end{array}$ & $\begin{array}{l}\text { Influence of Pharmaceutical Care } \\
\text { intervention and communication } \\
\text { skills on the improvement of } \\
\text { pharmacotherapeutic outcomes with } \\
\text { older adult Brazilian outpatients. }\end{array}$ & $\begin{array}{l}\text { Semi- } \\
\text { experimental }\end{array}$ & $\begin{array}{l}30 \text { (between } 60 \text { and } \\
75 \text { years old), Basic } \\
\text { Health Unit }\end{array}$ & 12 months & Hypertension \\
\hline $\begin{array}{l}\text { Lyra-Júnior, } \\
\text { Marcellin, Pelá, } \\
2008 \\
\text { (Ribeirão Preto, } \\
\text { São Paulo) }\end{array}$ & $\begin{array}{l}\text { Effect of pharmaceutical care } \\
\text { intervention on blood pressure } \\
\text { of older adult outpatients with } \\
\text { hypertension. }\end{array}$ & $\begin{array}{l}\text { Semi- } \\
\text { experimental }\end{array}$ & $\begin{array}{l}30 \text { (between } 60 \text { and } \\
75 \text { years old), Basic } \\
\text { Health Unit }\end{array}$ & 12 months & Hypertension \\
\hline $\begin{array}{l}\text { Obreli-Neto } \\
\text { et al., 2011a } \\
\text { (Salto Grande, } \\
\text { São Paulo) }\end{array}$ & $\begin{array}{l}\text { Effect of a } 36 \text {-month pharmaceutical } \\
\text { care program on pharmacotherapy } \\
\text { adherence in older adult diabetic } \\
\text { and hypertensive patients. }\end{array}$ & $\begin{array}{l}\text { Randomized } \\
\text { controlled } \\
\text { clinical trial }\end{array}$ & $\begin{array}{l}\text { Control Group: } 97 ; \\
\text { Intervention Group: } \\
97 \text { (> } 60 \text { years old), } \\
\text { Basic Health Unit }\end{array}$ & 36 months & $\begin{array}{l}\text { Hypertension } \\
\text { and/or } \\
\text { diabetes } \\
\text { mellitus }\end{array}$ \\
\hline $\begin{array}{l}\text { Obreli-Neto } \\
\text { et al., 2011b } \\
\text { (Salto Grande, } \\
\text { São Paulo) }\end{array}$ & $\begin{array}{l}\text { Effect of a 36-Month Pharmaceutical } \\
\text { Care Program on Coronary Heart } \\
\text { Disease Risk in Older adult Diabetic } \\
\text { and Hypertensive Patients. }\end{array}$ & $\begin{array}{l}\text { Randomized } \\
\text { controlled } \\
\text { clinical trial }\end{array}$ & $\begin{array}{l}\text { Control Group: } 97 ; \\
\text { Intervention Group: } \\
97 \text { (> } 60 \text { years old), } \\
\text { Basic Health Unit }\end{array}$ & 36 months & $\begin{array}{l}\text { Hypertension } \\
\text { and/or } \\
\text { diabetes } \\
\text { mellitus }\end{array}$ \\
\hline $\begin{array}{l}\text { Obreli-Neto } \\
\text { et al., } 2015 \\
\text { (Salto Grande, } \\
\text { São Paulo) }\end{array}$ & $\begin{array}{l}\text { Economic Evaluation of a } \\
\text { Pharmaceutical Care Program for } \\
\text { Older adult Diabetic and Hypertensive } \\
\text { Patients in Primary Health Care: } \\
\text { A } 36 \text {-Month Randomized Controlled } \\
\text { Clinical Trial. }\end{array}$ & $\begin{array}{l}\text { Randomized } \\
\text { controlled } \\
\text { clinical trial }\end{array}$ & $\begin{array}{l}\text { Control Group: } 97 ; \\
\text { Intervention Group: } \\
97 \text { (> } 60 \text { years old), } \\
\text { Basic Health Unit }\end{array}$ & 36 months & $\begin{array}{l}\text { Hypertension } \\
\text { and/or } \\
\text { diabetes } \\
\text { mellitus }\end{array}$ \\
\hline $\begin{array}{l}\text { Reinhardt et al., } \\
2012 \\
\text { (Novo Hamburgo, } \\
\text { Rio Grande do Sul) }\end{array}$ & $\begin{array}{l}\text { Pharmacotherapeutic monitoring } \\
\text { in hypertensive eldery living in a } \\
\text { geriatric home in Vale dos Sinos } \\
\text { region, Rio Grande do Sul State, Brazil. }\end{array}$ & $\begin{array}{l}\text { Observational } \\
\text { study with } \\
\text { longitudinal } \\
\text { retrospective } \\
\text { design }\end{array}$ & $\begin{array}{l}31 \text { (> } 60 \text { years old), } \\
\text { Geriatric nursing } \\
\text { home }\end{array}$ & 23 months & Hypertension \\
\hline $\begin{array}{l}\text { Viana, Arantes, } \\
\text { Ribeiro, } 2017 \\
\text { (São Paulo, } \\
\text { São Paulo) }\end{array}$ & $\begin{array}{l}\text { Interventions of the clinical } \\
\text { pharmacist in na Intermediate Care } \\
\text { Unit for older adult patients. }\end{array}$ & $\begin{array}{l}\text { Prospective } \\
\text { descriptive } \\
\text { study }\end{array}$ & $\begin{array}{l}80 \text { (>60 years old), } \\
\text { Hospital }\end{array}$ & $\begin{array}{l}\text { From } 24 \\
\text { hours after } \\
\text { admission } \\
\text { to hospital } \\
\text { discharge, } \\
\text { transfer or } \\
\text { death. }\end{array}$ & $\begin{array}{l}\text { Need for } \\
\text { critical care }\end{array}$ \\
\hline
\end{tabular}


Table 4. Aim, clinical services, indicators, and outcomes of the included articles $(n=11)$.

\begin{tabular}{|c|c|c|c|}
\hline $\begin{array}{l}\text { Author, year } \\
\text { (origin) }\end{array}$ & Aim & $\begin{array}{l}\text { Pharmaceutical clinical } \\
\text { services performed }\end{array}$ & Outcomes \\
\hline $\begin{array}{l}\text { Aguiar et al., } 2012 \\
\text { (Aracajú, Sergipe) }\end{array}$ & $\begin{array}{l}\text { Evaluate the effect of a pilot } \\
\text { pharmaceutical care program } \\
\text { developed for older adult patients } \\
\text { with uncontrolled hypertension. }\end{array}$ & $\begin{array}{l}\text { Pharmacotherapeutic } \\
\text { follow-up (monthly) } \\
\text { and assessment of } \\
\text { medication adherence. }\end{array}$ & $\begin{array}{l}57.2 \% \text { achieved blood pressure control }(p=0.000) \\
\text { and the mean reduction was } 26.6 \mathrm{mmHg}(p<0.0001) \\
\text { for systolic blood pressure, } 10.4 \mathrm{mmHg}(p<0.0001) \\
\text { for diastolic blood pressure, and } 15.7 \mathrm{mmHg}(p< \\
0.0001) \text { for pulse pressure. Medication adherence } \\
\text { also improved ( } p=0.0000) \text {. Anthropometric } \\
\text { measurements remained unchanged. }\end{array}$ \\
\hline $\begin{array}{l}\text { Brito et al., } \\
2009 \\
\text { (Aracajú, Sergipe) }\end{array}$ & $\begin{array}{l}\text { Evaluate the effect of a } \\
\text { pharmacotherapeutic management } \\
\text { program in a group of older adult } \\
\text { people with hypertension assisted } \\
\text { in a primary health care unit. }\end{array}$ & $\begin{array}{l}\text { Pharmacotherapeutic } \\
\text { follow-up (monthly to } \\
\text { quarterly). }\end{array}$ & $\begin{array}{l}\text { There was a reduction in consumption of NSAID } \\
(25.3 \% \text { to } 10 \% \text { ) and polypharmacy (from nine to six } \\
\text { patients). }\end{array}$ \\
\hline $\begin{array}{l}\text { Lyra-Júnior et al., } \\
2005 \text { (Ribeirão } \\
\text { Preto, São Paulo) }\end{array}$ & $\begin{array}{l}\text { Draw the socio-demographic } \\
\text { profile of a group of aged to } \\
\text { implement pharmaceutical } \\
\text { care; analyze the outcomes of } \\
\text { pharmacist's interventions; assess } \\
\text { satisfaction. }\end{array}$ & $\begin{array}{l}\text { Pharmacotherapeutic } \\
\text { follow-up (monthly). }\end{array}$ & $\begin{array}{l}91 \text { DRP were identified ( } 3.0 \pm 1.7 \mathrm{DRP} / \text { patient) and } \\
\text { pharmaceutical interventions solved about } 70 \% \text { of } \\
\text { them. There was a significant reduction in mean } \\
\text { systolic blood pressure }(18 \mathrm{mmHg}) \text { and diastolic } \\
\text { blood pressure }(12 \mathrm{mmHg}) \text {. Satisfaction showed high } \\
\text { scores. }\end{array}$ \\
\hline $\begin{array}{l}\text { Lyra-Júnior et al., } \\
\text { 2007a (Ribeirão } \\
\text { Preto, São Paulo) }\end{array}$ & $\begin{array}{l}\text { Evaluate the impact of } \\
\text { Pharmaceutical Care in } \\
\text { identification and resolution of } \\
\text { DRP and in quality of life of older } \\
\text { adults outpatients with chronic } \\
\text { conditions. }\end{array}$ & $\begin{array}{l}\text { Pharmacotherapeutic } \\
\text { follow-up (monthly). }\end{array}$ & $\begin{array}{l}92 \text { DRP were identified ( } 3.0 \pm 1.5 \text { DRP per patient), } \\
\text { with interventions solving } 69 \% \text { of actual DRP and } \\
\text { preventing } 78.5 \% \text { of potential DRP. Quality of life } \\
\text { showed improvement in } 22 \text { patients. }\end{array}$ \\
\hline $\begin{array}{l}\text { Lyra-Júnior et al., } \\
\text { 2007b (Ribeirão } \\
\text { Preto, São Paulo) }\end{array}$ & $\begin{array}{l}\text { Evaluate the influence of } \\
\text { Pharmaceutical Care intervention } \\
\text { on the results obtained with } \\
\text { a older adults outpatients and } \\
\text { analyze communication skills } \\
\text { of health professionals during } \\
\text { counseling about healthcare and } \\
\text { drugs. }\end{array}$ & $\begin{array}{l}\text { Pharmacotherapeutic } \\
\text { follow-up (monthly). }\end{array}$ & $\begin{array}{l}590 \text { interventions were performed ( } 376 \text { on health } \\
\text { education), } 64 \% \text { of them required physician } \\
\text { acceptability, which occurred in } 86 \% \text { of the cases. } \\
\text { There was an average of } 4+/-2 \text { interventions to } \\
\text { solve each DRP. } 93 \% \text { of patients reported that the } \\
\text { research pharmacist always gave them guidance for } \\
\text { the use of drugs and } 96 \% \text { stated that the research } \\
\text { pharmacist showed interest in clarifying their doubts. }\end{array}$ \\
\hline $\begin{array}{l}\text { Lyra-Júnior, } \\
\text { Marcellini, Pelá, } \\
2008 \\
\text { (Ribeirão Preto, } \\
\text { São Paulo) }\end{array}$ & $\begin{array}{l}\text { Evaluate the effect of pharmacist } \\
\text { intervention on the prevention } \\
\text { and solution of DRP, BMI, } \\
\text { and blood pressure control in } \\
\text { older adult outpatients with } \\
\text { hypertension. }\end{array}$ & $\begin{array}{l}\text { Pharmacotherapeutic } \\
\text { follow-up (monthly). }\end{array}$ & $\begin{array}{l}92 \mathrm{DRP} \text { were identified and } 590 \text { interventions were } \\
\text { carried out. Patients had a } 27 \% \text { reduction in weight } \\
\text { (weight loss greater than } 7 \% \text { ), but there was no } \\
\text { difference in BMI. The mean reductions in systolic } \\
\text { blood pressure }(18 \mathrm{mmHg}) \text { and diastolic blood } \\
\text { pressure }(12 \mathrm{mmHg} \text { ) were significant }(p<0.01) \text {. }\end{array}$ \\
\hline $\begin{array}{l}\text { Obreli-Neto } \\
\text { et al., 2011a } \\
\text { (Salto Grande, São } \\
\text { Paulo) }\end{array}$ & $\begin{array}{l}\text { Evaluate the effect of a } \\
\text { pharmaceutical care program } \\
\text { on pharmacotherapy adherence } \\
\text { in older adult diabetic and } \\
\text { hypertensive patients. }\end{array}$ & $\begin{array}{l}\text { Pharmacotherapeutic } \\
\text { follow-up (half-yearly), } \\
\text { educational activities } \\
\text { with groups of patients } \\
\text { and assessment of } \\
\text { pharmacotherapy } \\
\text { adherence. }\end{array}$ & $\begin{array}{l}\text { Significant improvements ( } p<0.001) \text { in Morisky- } \\
\text { Green score (adherent: } 49 \text { vs. } 81 \%) \text { and in the } \\
\text { dispensed medication history (adherent: } 51 \text { vs. } \\
81 \%) \text {; significant improvements }(p<0.05) \text { in the } \\
\text { frequency of patients with controled blood pressure } \\
(26 \text { vs. } 84 \%) \text {, glycemic index (fasting glucose: } 29 \text { vs. } \\
68 \%, \text { A1C hemoglobin: } 1 \text { vs. } 19 \%) \text { and lipid levels } \\
\text { (triglycerides: } 46 \text { vs. } 72 \% \text {; total cholesterol: } 58 \text { vs. } \\
78 \% \text { ). }\end{array}$ \\
\hline $\begin{array}{l}\text { Obreli-Neto } \\
\text { et al., 2011b } \\
\text { (Salto Grande, São } \\
\text { Paulo) }\end{array}$ & $\begin{array}{l}\text { Examine the effect of an } \\
\text { implemented pharmaceutical care } \\
\text { program on coronary heart disease } \\
\text { risk in older adult diabetic and } \\
\text { hypertensive patients. }\end{array}$ & $\begin{array}{l}\text { Pharmacotherapeutic } \\
\text { follow-up (half-yearly), } \\
\text { educational activities } \\
\text { with groups of patients } \\
\text { and assessment of } \\
\text { pharmacotherapy } \\
\text { adherence. }\end{array}$ & $\begin{array}{l}\text { Significant reductions }(p<0.001) \text { in systolic blood } \\
\text { pressure values }(156.7 \mathrm{vs} .133 .7 \mathrm{mmHg}) \text {, diastolic } \\
\text { blood pressure }(106.6 \mathrm{vs} .91 .6 \mathrm{mmHg}) \text {, fasting } \\
\text { glucose }(135.1 \text { vs. } 107.9 \mathrm{mg} / \mathrm{dL}), \mathrm{A} 1 \mathrm{C} \text { hemoglobin } \\
(7.7 \mathrm{vs} .7 .0 \%) \text {, triglycerides }(206.0 \mathrm{vs} .152 .5 \mathrm{mg} / \\
\mathrm{dL}), \mathrm{LDL}(112.4 \mathrm{vs} .102 .0 \mathrm{mg} / \mathrm{dL}), \mathrm{HDL}(55.5 \mathrm{vs} .65 .5 \\
\mathrm{mg} / \mathrm{dL}), \text { total cholesterol }(202.5 \mathrm{vs} .185 .9 \mathrm{mg} / \mathrm{dL}) \text {, } \\
\text { BMI }(26.2 \text { vs. } 26.1 \mathrm{~kg} / \mathrm{m} 2) \text {, abdominal circumference } \\
(103.2 \text { vs. } 102.5 \mathrm{~cm}) \text { and cardiovascular risk (6.8 vs. } \\
4.8 \%) \text {. }\end{array}$ \\
\hline
\end{tabular}




\section{lutarma}

\begin{tabular}{|c|c|c|c|}
\hline $\begin{array}{l}\text { Author, year } \\
\text { (origin) }\end{array}$ & Aim & $\begin{array}{l}\text { Pharmaceutical clinical } \\
\text { services performed }\end{array}$ & Outcomes \\
\hline $\begin{array}{l}\text { Obreli-Neto } \\
\text { et al., } 2015 \\
\text { (Salto Grande, São } \\
\text { Paulo) }\end{array}$ & $\begin{array}{l}\text { Evaluate the economic cost } \\
\text { and the ICER per QALY of } \\
\text { pharmaceutical care in the } \\
\text { management of diabetes and } \\
\text { hypertension in older adult } \\
\text { patients in a primary public } \\
\text { health care system in a } \\
\text { developing country. }\end{array}$ & $\begin{array}{l}\text { Pharmacotherapeutic } \\
\text { follow-up (half-yearly), } \\
\text { educational activities } \\
\text { with groups of patients } \\
\text { and assessment of } \\
\text { pharmacotherapy } \\
\text { adherence. }\end{array}$ & $\begin{array}{l}\text { No statistically significant difference was found } \\
\text { in total direct health care costs }(p=0.089) \text {; } \\
\text { pharmaceutical care added incremental costs of } \\
\$ 69.60( \pm \$ 7.90) \text { per patient. The ICER per QALY was } \\
\$ 53.50(95 \% \text { CI }=\$ 51.60-54.00) \text {. Every clinical } \\
\text { parameter evaluated improved for the pharmaceutical } \\
\text { care group. }\end{array}$ \\
\hline $\begin{array}{l}\text { Reinhardt } \\
\text { et al., } 2012 \\
\text { (Novo Hamburgo, } \\
\text { Rio Grande do Sul) }\end{array}$ & $\begin{array}{l}\text { Evaluate the pharmacotherapy } \\
\text { in hypertensive older adult } \\
\text { living in a geriatric home, after } \\
\text { pharmacotherapeutic monitoring } \\
\text { and pharmaceutical interventions. }\end{array}$ & $\begin{array}{l}\text { Pharmacotherapeutic } \\
\text { follow-up (with no } \\
\text { periodicity specified). }\end{array}$ & $\begin{array}{l}\text { There was no significant change in diastolic blood } \\
\text { pressure }(p=0.148) \text {, however, there was a decrease } \\
\text { in systolic blood pressure }(p<0.001) \text { and in mean } \\
\text { blood pressure }(p=0.002) \text { of all participants. }\end{array}$ \\
\hline $\begin{array}{l}\text { Viana, Arantes, } \\
\text { Ribeiro, } 2017 \text { (São } \\
\text { Paulo, } \\
\text { São Paulo) }\end{array}$ & $\begin{array}{l}\text { Discuss the role of the clinical } \\
\text { pharmacist in hospital care of } \\
\text { critical older adult patients. }\end{array}$ & $\begin{array}{l}\text { Pharmacotherapy review } \\
\text { (daily); therapeutic drug } \\
\text { monitoring. }\end{array}$ & $\begin{array}{l}212 \text { interventions were performed in } 62 \text { patients } \\
(77.5 \%) \text {, with an average of } 3 \text { interventions per } \\
\text { patient. } 64.3 \% \text { of the interventions were accepted, } \\
28.5 \% \text { were not accepted and } 7.2 \% \text { were verbally } \\
\text { accepted, but without changes in the prescription. }\end{array}$ \\
\hline
\end{tabular}

As the included studies have different designs and populations (elderly people with specific health problems, at different levels of health care), it was impossible to perform meta-analysis or other quantitative analyses to summarize the results. However, the qualitative analysis gives us an overview of the outcomes of pharmaceutical care for the elderly in Brazil.

Unfortunately, in Brazil, there is a plurality of concepts of pharmaceutical care, clinical pharmacy, and clinical pharmacy services, especially when we consider changes in definitions that have happened over the years. Therefore, in an attempt to cover all articles published, even with the conceptual variations, the elaboration of the search strategy considered several terms that could refer to pharmaceutical care, including the term "pharmacist." This fact justifies the high number of articles obtained at first, which were screened by reading for those that portrayed pharmaceutical care.

The global scientific literature has highlighted the importance of pharmaceutical care for older adult patients with different health problems in various scenarios, such as hospitals, geriatric nursing homes, community pharmacies, and outpatient clinics $(9,26,27)$. However, in preparation for this systematic review, we did not obtain many publications addressing this issue in Brazil. Moreover, the included articles referring to Ribeirão Preto and
Salto Grande evaluate different variables resulting from the same research and thus, they report results of pharmaceutical care in the same place and period. Therefore, it can be stated that this systematic review obtained data resulting from the implementation of pharmaceutical care in six different situations, covering five different municipalities in three regions of the country (southeast, northeast, and South).

The low number of publications is indicative of the more recent implementation of pharmaceutical care in Brazil, which became discussed and effectively performed only in the last decade. The first official discussion on pharmaceutical care in Brazil began in the second half of the 1990s, led by the Pan American Health Organization (PAHO) (28). The clinical training process during pharmacy graduation was not adequate, representing an important barrier to pharmaceutical care in the country. In 2017, the Federal Pharmacy Council published the document entitled "Competencies for the Pharmacist Clinical Performance," which resulted in the elaboration of a new curriculum matrix aimed as a guiding instrument for the clinical training of the pharmacists in the country (29-31). In Europe and North America, the pharmacist clinical services are well established, with a robust training program in this area in the universities (28).

The small number of participants included in the studies indicates that these services are new and 
do not yet have pharmacists dedicated exclusively to performing clinical services.

The first Brazilian publication dealing with pharmaceutical care in elder patients was published in 2005, with the data collection in 2003 (20). Thus, the beginning of the publications coincides with the first experiences with pharmaceutical care in Brazil. Although the use of drugs is a relevant topic in all age groups, research on this subject has often been focused on older adult patients owing to the peculiar characteristics of this population, such as comorbidities, polypharmacy, complex therapies, pharmacokinetic and pharmacodynamic variations, loss of functional capacity, difficulties in adherence, errors in administration, cognitive disorders, visual difficulty, and impaired manual dexterity (32). This is the basis for the initiating services focused on older adult patients in the first experiment involving pharmaceutical care in the country.

Regarding the methodological quality, most studies were of medium or poor quality. The methodological design and the levels of evidence of the studies were the determinants of the quality score, with randomized clinical trials being of the highest quality $(18,19,22)$, followed by interventional studies $(16,17,20,21)$. The descriptive articles received a lower score $(5,25)$, and only one article on the interventional study was classified as poor quality (24). The absence of studies of excellence may also reflect the difficulty of Brazilian researchers in obtaining financial resources for science, which limits the study's design and interferes with the quality (33). The small number of studies obtained and the absence of studies with more robust designs and a low level of bias make it difficult to carry out reliable comparisons and conclusions and perform quantitative analyses. However, the qualitative analysis allowed us to overview pharmaceutical care for the elderly in Brazil and how much it still needs to be expanded and improved.

Hypertension and type 2 diabetes mellitus (DM2) are highly prevalent health problems and are recognized as risk factors for cardiovascular disease and morbidity and mortality in the older adult population (34). In Brazil, hypertension and DM2 in older adult individuals are around 50\% and $20 \%$, respectively (34-36). In addition, over $60 \%$ of the patients with hypertension and $40 \%$ with DM2 fail to achieve control of the disease (37-39), which makes the application of pharmaceutical care in a collaborative and multi-professional work context a potential tool to improve the control indexes, and thus, reducing cardiovascular complications. These notes explain the predominance of participants with hypertension and DM2 in the articles included in this review.

It should be noted that six of the included publications evaluated the primary outcomes of the service, i.e., the outcomes that could effectively demonstrate the clinical improvement of the patient, such as the reduction of the glycemic index, blood pressure, lipid levels, and anthropometric measurements $(18-21,23,25)$. The other articles were selected to evaluate the secondary clinical outcomes (pharmacotherapy adherence, number of DRP found, number and acceptability of pharmaceutical interventions, and reduction in the number of drugs in use), or humanistic outcomes (quality of life and satisfaction), which are positively impacted by the pharmaceutical care and are more easily measurable.

Only one of the included publications sought to analyze the economic impact of the pharmaceutical care of older adult patients (22). The treatment of health problems and complications in more advanced age groups generally requires more complex and expensive technologies; therefore, implementing measures that can reduce the complications and promote the rational use of drugs can have a favorable economic impact on the health system $(22,32,40)$. This study evaluated the economic cost and the incremental cost-effectiveness ratio (ICER) per quality-adjusted life-year (QALY) of pharmaceutical care. Pharmaceutical care did not significantly increase total direct health care costs, and significantly improved health outcomes were seen, so the mean ICER per QALY gained suggests favorable cost-effectiveness (22). Analyzing economic outcomes associated with the clinical outcomes of pharmaceutical care should be encouraged in Brazil, especially when we consider that the Brazilian health care model is public and universal.

Although a small number of publications that do not represent the highest levels of evidence were obtained, all these studies confirmed the expected benefit of pharmaceutical care in the care of older 
adult patients, regardless of the level of healthcare. It should be noted that pharmaceutical care was an effective tool in reducing the blood pressure, glycemic index, and lipid levels in all the selected studies to evaluate these variables $(18,20,21,23,25)$. There was no benefit in reducing the anthropometric measurements as observed in two studies $(21,23)$, but a third publication demonstrated a reduction in body mass index and abdominal circumference (19). In addition to improving the quality of life and adherence to pharmacotherapy $(16,18,23)$, pharmaceutical care has also been proven useful in reducing polypharmacy (24) and solving DRP $(5,16,17,20,21)$.

Older adult patients have recognized the benefits of pharmaceutical care as they are more satisfied with the health service (20). The high acceptability of pharmaceutical interventions by the health care team, as reported in some publications, attests to the recognition by other professionals of the contributions of pharmaceutical care $(5,17)$.

This activity of the pharmacists has also been reflected in favorable cost-effectiveness as it did not significantly increase the overall health costs and resulted in improvement in several clinical outcomes (22).

Through this review, it was possible to identify and summarize the evidence supporting pharmaceutical clinical services' performance for older adult patients in Brazil, especially in pharmacotherapeutic follow-up, pharmacotherapy review, and educational activities.

The fact that it does not cover the gray literature, i.e., it does not include the unpublished productions in the form of scientific articles, is a limitation of this systematic review. Since many clinical pharmacists do not publish their results, this review may underestimate the scope of pharmaceutical care for older adult patients in Brazil. This may reflect a lack of publishing culture among the pharmacists and even Brazil's Unique Health System (SUS) workers. It is essential to make such professionals aware of the importance of recording and disseminating the results of clinical services. Since it is a relatively new professional activity in Brazil and is little known to the people and other healthcare professionals, the dissemination may help create demand and encourage the expansion of pharmaceutical care in the country. The lack of standardization in studies evaluating clinical pharmaceutical services was also a limitation since the differences in design made it impossible to perform quantitative analyzes.

Although it has limitations, like any other study, it is important to emphasize that this systematic review makes important contributions toward the evolution of the state of the art of pharmaceutical care for older adult patients, emphasizing that pharmaceutical care is an important tool for improving the quality of life of these patients and for the health system as a whole. Pharmaceutical care in the elderly can benefit the patient with improved quality of life, safety in treatment, and improved clinical outcomes. The benefits extend to the health system, which improves the humanization of the services offered and avoids worsening illnesses and prolonged hospitalizations from an economic perspective. However, this is still not a routine activity at any level of healthcare in Brazil. This warns of the need to institute health policies that encourage the implementation of pharmaceutical care across the country at all levels of healthcare. To meet this demand, institutions such as the Ministry of Health, National Council of Municipal Health Secretaries, universities, and the Federal Pharmacy Council have improved pharmacists' knowledge and clinical skills through in-service training. As a result, it is expected that the reality will be different from that reported in this review, with a wider range of quality scientific articles discussing the subject within a few years.

\section{CONCLUSION}

This systematic review indicates that the experiences of older adult Brazilians who were provided pharmaceutical care contributed to improved clinical, humanistic, and economic outcomes, especially through pharmacotherapeutic follow-up, pharmacotherapy review and educational activities. The pharmacist presented a favorable clinical performance in different scenarios and levels of healthcare. However, only a limited number of pharmacists were committed to recording and dis- 
seminating the results in the scientific literature. For pharmaceutical care in this population to be amplified, benefiting patients and the health system, it is essential to conduct more robust studies emphasizing the importance and contribution of this activity in all regions of Brazil. Moreover, considering the recent nature of the pharmacist's clinical activities in the country, it is important to ensure proper training of these professionals to acquire skills, abilities, knowledge, and appropriate behaviors.

\section{ACKNOWLEDGMENT}

The authors disclosed receipt of the following financial support for the research, authorship, and/or publication of this article: this work was supported by the Brazilian National Council for Scientific and Technological Development - CNPq (Social Demand Program PhD scholarship, grant number 142154/2016-4).

\section{REFERENCES}

1. Veras R. Can growing old in Brazil involve good health and quality of life? Rev. bras. geriatr. gerontol. 2016;19(3):381-382. DOI: 10.1590/1809-98232016019. 160100 .

2. IBGE. Projection of the population of Brazil and the Federation Units. Brazilian Institute of Geography and Statistics. Available in: https:/www.ibge.gov.br/apps/ populacao/projecao/. Accessed on: November 06, 2020.

3. Lyra-Júnior DP, Amaral RT, Veiga EV, Cárnio EC, Nogueira MS, Pelá IR. A farmacoterapia no idoso: revisão sobre a abordagem multiprofissional no controle da Hipertensão Arterial Sistêmica. Rev Latino-am Enferm. 2006; 14(3):435-441. DOI:10.1590/S010411692006000300019 .

4. Kane RL, Ouslander JG, Abrass IB, Resnick B. Fundamentos de geriatria clínica. 7th ed. Porto Alegre: Porto Alegre: McGraw Hill; 2015.

5. Viana SSC, Arantes T, Ribeiro SCC. Interventions of the clinical pharmacist in an Intermediate Care Unit for older adult patients. Einstein. 2017;15(3):283-288. DOI: 10.1590/S1679-45082017AO3894.

6. Medeiros EFF, Moraes CF, Karnikowski M, Nóbrega OT, de Oliveira Karnikowski MG. Intervenção interdisciplinar enquanto estratégia para o Uso Racional de Medicamentos em idosos. Cienc e Saude Colet. 2011;16:3139-3149. DOI: 10.1590/S1413-8123201100 0800014

7. Fick DM, Semla TP, Steinman M, Beizer J, Brandt N, Dombrowski R, et al. American Geriatrics Society 2019 Updated AGS Beers Criteria ${ }^{\circledR}$ for Potentially Inappropriate Medication Use in Older Adults. J Am Geriatr Soc. 2019;67:674-694. DOI: 10.1111/jgs.15767.

8. Oliveira MG, Amorim WW, Oliveira CRB, Coqueiro HL, Gusmão LC, Passos LC. Consenso brasileiro de medicamentos potencialmente inapropriados para idosos. Geriatr Gerontol Aging. 2017;10:168-181. DOI: 10.5327/Z2447-211520161600054.
9. Sáez-Benito L, Fernandez-Llimos F, Feletto E, Gastelurrutia MA, Martinez-Martinez F, Benrimoj SI. Evidence of the clinical effectiveness of cognitive pharmaceutical services for aged patients. Age Ageing 2013;42:442-449. DOI: 10.1093/ageing/aft045.

10. Moher D, Shamseer L, Clarke M, Ghersi D, Liberatî A, Petticrew M, et al. Preferred reporting items for systematic review and meta-analysis protocols (PRISMA-P) 2015 statement. Syst Rev. 2015, 4:1-9. DOI: 10.1186/20464053-4-1.

11. Santos CMC, Pimenta CAM, Nobre MRC. A estratégia PICO para a construção da pergunta de pesquisa e busca de evidências. Rev Latino-am Enferm. 2007; 15(3):508511. DOI:10.1590/S0104-11692007000300023.

12. Ouzzani M, Hammady H, Fedorowicz Z, et al. Rayyan a web and mobile app for systematic reviews. Syst Rev. 2016;5(210):1-10. DOI: 10.1186/s13643-016-0384-4.

13. Higgins JPT, Green S. Cochrane handbook for systematic reviews of interventions version 5.1.0. The Cochrane Collaboration, 2011. [citad 2020 Nov 02]. Available from: http://handbook.cochrane.org.Higgins

14. Downs S, Black N. The feasibility of creating a checklist for the assessment of the methodological quality both of randomized and non-randomized studies of health care interventions. J Epidemiol Community Heal. 1998;52:377-384. DOI: 10.1136/jech.52.6.377

15. O 'Connor SR, Tully MA, Ryan B, Bradley JM, Baxter GD, Mcdonough SM. Failure of a numerical quality assessment scale to identify potential risk of bias in a systematic review: a comparison study. BMC Res Notes. 2015;8 (224):1-7. DOI: 10.1186/s13104-015-1181-1.

16. Lyra-Júnior DP, Kheir N, Abriata JP, Rocha CE, Santos CB, Pelá IR. Impact of Pharmaceutical Care interventions in the identification and resolution of drug-related problems and on quality of life in a group of elderly outpatients in Ribeirão Preto (SP), Brazil. Ther Clin Risk Manag. 2007;3(6):989-998. 
17. Lyra-Júnior DP, Rocha CE, Abriata JP, Gimenes FRE, Gonzalez MM, Pelá IR. Influence of Pharmaceutical Care intervention and communication skills on the improvement of pharmacotherapeutic outcomes with elderly Brazilian outpatients. Patient Educ Couns. 2007;68(2):186-192. DOI: 10.1016/j.pec.2007.06.004.

18. Obreli-Neto PR, Guidoni CM, Baldoni AO, Pilger D, Cruciol-Souza JM, Gaeti-Franco WP, Cuman RKN. Effect of a 36-month pharmaceutical care program on pharmacotherapy adherence in elderly diabetic and hypertensive patients. Int J Clin Pharm. 2011;33(4):642649. DOI: 10.1007/s11096-011-9518-x.

19. Obreli-Neto PR, Marusic S, Lyra-Júnior DP, Pilger D, Cruciol-Souza JM, Gaeti WP, Cuman RKN. Effect of a 36-month pharmaceutical care program on coronary heart disease risk in elderly diabetic and hypertensive patients. J Pharm Pharm Sci. 2011;14(2):249-263. DOI: $10.18433 / \mathrm{j} 3259 \mathrm{q}$.

20. Lyra-Júnior D, Amaral RT, Abriata JP, Pelá IR. Satisfacción como resultado de un programa de atención farmacéutica para pacientes ancianos en Ribeirão Preto São Paulo (Brasil). Seguim Farmacoter. 2005;3(1):30-42.

21. Lyra Júnior DP, Marcellini PS, Pelá IR. Effect of pharmaceutical care intervention on blood pressure of older adult outpatients with hypertension. Bras J Pharm Sci. 2008;44(3):451-457. DOI: 10.1590/S151693322008000300015 .

22. Obreli-Neto PR, Marusic S, Guidoni CM, Baldoni AO, Renovato RD, Pilger D, Cuman RKN, Pereira LRL. Economic evaluation of a pharmaceutical care program for elderly diabetic and hypertensive patients in primary health care: A 36-month randomized controlled clinical trial. J Manag Care Pharm. 2015;21:66-75. DOI: 10.18553/jmcp.2015.21.1.66.

23. Aguiar PM, Balisa-Rocha BJ, Brito GC, Lyra-Júnior DP. Pharmaceutical care program for older adult patients with uncontrolled hypertension. J Am Pharm Assoc. 2012;52(4):515-518. DOI: 10.1331/JAPhA.2012.11015.

24. Brito GC, Menezes MS, Mesquita AR, Lyra-Júnior DP. Efeito de um programa de manejo farmacoterapêutico em um grupo de idosos com hipertensão em Aracaju-Sergipe. Rev Ciencias Farm Basica e Apl. 2009;30:83-89.

25. Reinhardt F, Ziulkoski AL, Andrighetti LH, Perassolo MS. Acompanhamento farmacoterapêutico em idosos hipertensos residentes em um lar geriátrico, localizado na Região do Vale dos Sinos, Rio Grande do Sul, Brasil. Rev Bras Geriatr e Gerontol 2012;15:109-117. DOI: 10.1590/S1809-98232012000100012.

26. Chen JH, Ou HT, Lin TC, Lai ECC, Kao YHY. Pharmaceutical care of elderly patients with poorly controlled type 2 diabetes mellitus: a randomized controlled trial. Int J Clin Pharm. 2016;38:88-95. DOI: 10.1007/s11096-015-0210-4.
27. Lenssen R, Schmitz K, Griesel C, Heidenreich A, Schulz JB, Trautwein C, Marx N, Fitzner C, Jaehde U, Eisert A. Comprehensive pharmaceutical care to prevent drug-related readmissions of dependent-living elderly patients: a randomized controlled trial. BMC Geriatr. 2018;18(1):135-144. DOI: 10.1186/s12877-018-0814-3.

28. Pereira LRL, Freitas O. A evolução da Atenção Farmacêutica e a perspectiva para o Brasil. Braz J Pharm Sci. 2008;44:601-612.

29. Oliveira AB, Oyakawa CN, Miguel MD, Zanin SMW, Montrucchio DP. Obstáculos da atenção farmacêutica no Brasil. Braz. J. Pharm. Sci. 2005; 41(4):409-413.

30. Nicoline CB, Vieira RCPA. Assistência farmacêutica no sistema único de saúde (SUS): Percepções de graduandos em farmácia. Interface Commun Heal Educ. 2011;15:1127-1141. DOI: 10.1590/S141432832011005000026 .

31. BRASIL. Ministério da Educação. Resolução n ${ }^{\circ}$ 6, de 19 de outubro de 2017. Institui as Diretrizes Curriculares Nacionais do Curso de Graduação em Farmácia e dá outras providências. Diário Oficial da União, 19 de outubro de 2017.

32. Meneses ALL, Sá MLB. Atenção farmacêutica ao idoso: fundamentos e propostas. 2010;4:154-161.

33. Oliveira FS, Bonacelli MBM. Low Efficiency in the Use of Research and Development resources in Brazilian public research organizations: causal chains analysis.. REAd. 2019;25:62-95. DOI: 10.1590/14132311.257.94205.

34. Francisco PMSB, Segri NJ, Borim FSA, Malta DC. Prevalence of concomitant hypertension and diabetes in brazilian older adults: Individual and contextual inequalities. Cienc e Saude Colet. 2018;23:3829-3840. DOI: 10.1590/1413-812320182311.29662016.

35. Andrade SSA, Stopa SR, Brito AS, Chueri PS, Szwarcwald CL, Malta DC. Prevalência de hipertensão arterial autorreferida na população brasileira: análise da Pesquisa Nacional de Saúde, 2013. Epidemiol e Serviços Saúde. 2015;24:297-304. DOI: 10.5123/S167949742015000200012.

36. Iser BPM, Stopa SR, Chueiri PS, Szwarcwald CL, Malta DC, Monteiro HOC, Duncan BB, Schmidt MI. Prevalência de diabetes autorreferido no Brasil: resultados da Pesquisa Nacional de Saúde 2013. Epidemiol e Serviços Saúde. 2015;24:305-314. DOI: 10.5123/S1679-49742015000200013

37. Pereira M, Lunet N, Azevedo A, Barros H. Differences in prevalence, awareness, treatment and control of hypertension between developing and developed countries. J Hypertens. 2009;27:963-975. DOI: 10.1097/ hjh.0b013e3283282f65. 
38. Casagrande SS, Fradkin JE, Saydah SH, Rust KF, Cowie $\mathrm{CC}$. The prevalence of meeting A1C, blood pressure, and LDL goals among people with diabetes, 1988-2010. Diabetes Care. 2013;36:2271-2279. DOI: 10.2337/ dc12-2258.

39. Moreira GC, Cipullo JP, Martin JFV, Ciorlia LAS, Godoy MRP, Cesarino CB, Cordeiro JA, Lupino PL, Ciorlia G, Burdmann EA. Evaluation of the awareness, control and cost-effectiveness of hypertension treatment in a Brazilian city: populational study. J Hypertens. 2009;27:19001907. DOI: 10.1097/HJH.0b013e32832dd10f.
40. Cazarim MS, Nunes AA, Pereira LRL. Cost-consequence analysis of Pharmaceutical Care program for systemic arterial hypertension in the public health system in Brazil. Braz J Pharm Sci. 2017;53(3):e00217. DOI: 10.1590/s2175-97902017000300217. 